PROCEEDINGS OF THE

AMERICAN MATHEMATICAL SOCIETY

Volume 129, Number 8, Pages 2395-2402

S 0002-9939(01)05911-1

Article electronically published on January 18, 2001

\title{
POLYNOMIAL APPROXIMATION ON THREE-DIMENSIONAL REAL-ANALYTIC SUBMANIFOLDS OF $\mathbf{C}^{n}$
}

\author{
JOHN T. ANDERSON, ALEXANDER J. IZZO, AND JOHN WERMER
}

(Communicated by Steven R. Bell)

\begin{abstract}
It was once conjectured that if $A$ is a uniform algebra on its maximal ideal space $X$ and if each point of $X$ is a peak point for $A$, then $A=C(X)$. This peak point conjecture was disproved by Brian Cole in 1968. However, it was recently shown by Anderson and Izzo that the peak point conjecture does hold for uniform algebras generated by smooth functions on smooth twomanifolds with boundary. Although the corresponding assertion for smooth three-manifolds is false, we establish a peak point theorem for real-analytic three-manifolds with boundary.
\end{abstract}

\section{INTRODUCTION}

In this note we consider the problem of approximating arbitrary continuous functions on a compact subset $K$ of $n$-dimensional complex Euclidean space $\mathbf{C}^{n}$ by polynomials in the coordinate functions $z_{1}, \ldots, z_{n}$. Let $C(K)$ denote the space of all continuous complex-valued functions on $K$, with norm $\|g\|_{K}=\max \{|g(z)|$ : $z \in K\}$, and let $P(K)$ denote the closure of the set of polynomials in $C(K)$. One necessary condition for $P(K)=C(K)$ is that $K$ be polynomially convex, i.e., that $K$ coincide with its polynomially convex hull

$$
\hat{K}=\left\{z \in \mathbf{C}^{n}:|Q(z)| \leq\|Q\|_{K} \text { for every polynomial } Q\right\} .
$$

Another necessary condition is that $K$ contain no analytically imbedded disk, for otherwise elements of $P(K)$ are holomorphic on that disk. In the case $n=1$, there is a simple geometric condition that is equivalent to polynomial convexity: $K=\hat{K}$ if and only if $\mathbf{C} \backslash K$ is connected. Moreover, Lavrentieff's Theorem (1], p.12) states that in the plane, the two necessary conditions mentioned above are also sufficient: if $\mathbf{C} \backslash K$ is connected, and $K$ has no interior, then $P(K)=C(K)$.

In higher dimensions matters are more complicated. There is no simple geometric condition that is known to be equivalent to polynomial convexity. Moreover, examples can be given of polynomially convex sets $K$ with no analytic structure for which $P(K) \neq C(K)$ (see the introduction to [8]). When $K$ lies on a smooth real submanifold 1 of $\mathbf{C}^{n}$, an important role is played by the size and structure of the set $E$ of points at which the manifold has a complex tangent. For example, a result of Wermer [12] implies that if $M$ is the graph in $\mathbf{C}^{2}$ of a smooth function on

Received by the editors December 28, 1999.

2000 Mathematics Subject Classification. Primary 32E30; Secondary 46 J10.

${ }^{1}$ The term "submanifold of $\mathbf{C}^{n}$ " will mean a relatively closed submanifold of some open subset of $\mathbf{C}^{n}$. 
the disk $\{z \in \mathbf{C}:|z| \leq 1\}$, and $M$ is polynomially convex, then $P(M)=C(M)$ if $E$ has two-dimensional measure zero. Hörmander and Wermer [7] and NirenbergWells [9] established approximation results for polynomially convex compact subsets of totally real $(E=\emptyset)$ manifolds; Hörmander and Wermer, as well as O'Farrell, Preskenis and Walsh [10] also studied certain cases in which complex tangents exist. Their results (see section 2) will be important for our work.

The condition that $K$ be polynomially convex has a natural interpretation when $P(K)$ is studied as a uniform algebra: $\hat{K}$ is identified with the maximal ideal space $\mathcal{M}_{A}$ of the algebra $A=P(K)$. However, from this point of view, it is natural to replace assumptions on the complex tangent set with another necessary condition for $P(K)=C(K)$. We will demand that every point of $K$ be a peak point for the algebra $P(K)$. Thus we are led to study compact sets $K$ satisfying the following two conditions:

(i) $K=\hat{K}$.

(ii) For each $p \in K$, there exists $g \in P(K)$ with $g(p)=1$ and $|g|<1$ on $K \backslash\{p\}$.

With a general uniform algebra $A$ on a compact metric space $X$ replacing $P(K)$, and with (i) replaced by $\mathcal{M}_{A}=X$, it was once conjectured that together these two necessary conditions for $A=C(X)$ were also sufficient to imply $A=C(X)$. However, a counterexample to this "peak point conjecture" was produced by Brian Cole in his 1968 thesis (see the Appendix to [4, or 11], section 19). Additional counterexamples to the peak point conjecture have since been given in the context of polynomial and rational approximation in several complex variables. Here the peak point hypothesis arises naturally as follows: if $K$ is a compact subset of the boundary of a strictly pseudoconvex domain (for example, the unit ball $B_{n}$ ) in $\mathbf{C}^{n}$, then every point of $K$ is a peak point for any uniform algebra $A$ on $X$ that contains the polynomials. Richard Basener [3] constructed a counterexample to the peak point conjecture where $A=R(K)$ is the closure in $C(K)$ of the rational functions with poles off $K$, for a certain set $K \subset \partial B_{2}$, and Alexander Izzo [8] has constructed counterexamples in which $A$ is the algebra $P(X)$ for a certain set $X \subset \partial B_{3}$. Izzo's paper also includes an example of this type where $X$ is a smooth solid torus in $\partial B_{5}$. In a positive direction, Anderson and Izzo [2] have recently established the peak point conjecture for uniform algebras generated by smooth functions on a twomanifold (with boundary). Their work relies on methods of Michael Freeman [6], who studied algebras generated by smooth functions on a two-manifold, generalizing the work of Wermer on polynomially convex disks mentioned above. As a special case of the results of [2], one obtains the fact that if $K=M$ is a two-manifold in $\mathbf{C}^{n}$ satisfying (i) and (ii), then $P(M)=C(M)$. The purpose of this paper is to prove the following analogous result on approximation on three-manifolds:

Theorem 1.1. Let $\Sigma$ be a real-analytic three-dimensional submanifold of $\mathbf{C}^{n}$. Let $X$ be a compact subset of $\Sigma$ such that $\partial X$ (the boundary of $X$ relative to $\Sigma$ ) is a two-dimensional submanifold of class $C^{1}$. If $X$ satisfies conditions (i) and (ii) above, then $P(X)=C(X)$.

Remark 1 . We allow $\partial X$ to be disconnected, or empty.

Remark 2. We have the following immediate corollary of Theorem 1.1:

Corollary 1.2. Let $\Sigma$ be a real-analytic three-dimensional submanifold contained in the boundary of the unit ball of $\mathbf{C}^{n}$, with $\partial \Sigma$ a two-dimensional submanifold of class $C^{1}$. Then $P(\Sigma)=C(\Sigma)$ if and only if $\Sigma$ is polynomially convex. 
Izzo's example (of a polynomially convex smooth three-manifold lying in the boundary of the unit ball on which polynomial approximation fails) shows that Corollary 1.2 is false if "real-analytic" is replaced by " $C^{\infty}$ ", and that the result of Anderson and Izzo mentioned above cannot be extended to three-manifolds without additional assumptions. We will see below that the real-analyticity assumption together with the peak-point hypothesis (ii) places strong restrictions on the size of the complex tangent set. In Izzo's example, the set of points at which $M$ has a complex tangent has positive three-dimensional Hausdorff measure; this cannot happen for the complex tangent set of $X$ under the hypotheses of Theorem 1.1.

\section{Preliminaries}

The numbers (i) and (ii) will refer to the polynomial convexity and peak-point conditions on a compact set $K \subset \mathbf{C}^{n}$ as defined in the introduction. These properties taken together are inherited by closed subsets of $K$ :

Lemma 2.1. Let $K$ be a compact subset of $\mathbf{C}^{n}$ satisfying (i) and (ii). Then every closed subset $Y$ of $K$ also satisfies (i) and (ii).

Proof. Clearly $\hat{Y} \subset \hat{K}=K$. Suppose $p \in K \backslash Y$. By property (ii) there exists $g \in P(K)$ with $g(p)=1$ and $|g|<1-d$ on $Y$, for some $d>0$. If $Q$ is a polynomial with $\|Q-g\|_{K}<d / 2$, then $|Q(p)|>\|Q\|_{Y}$; hence $p \notin \hat{Y}$. Hence $\hat{Y}=Y$. Now fix $y \in Y$. Since $K$ satisfies (ii), we can choose $g \in P(K)$ with $g(y)=1$ and $|g|<1$ on $K \backslash\{y\}$. Then the restriction of $g$ to $Y$ lies in $P(Y)$, and so (ii) is satisfied, with $K$ replaced by $Y$.

It is useful to know that adjoining a point to a polynomially convex set does not destroy polynomial convexity. This follows easily from [11], Lemma 29.21(b), but a direct proof is easily given.

Lemma 2.2. If $K \subset \mathbf{C}^{n}$ is polynomially convex and $z_{0} \in \mathbf{C}^{n}$, then $K \cup\left\{z_{0}\right\}$ is also polynomially convex.

Proof. Assume $z_{0} \notin K$, since otherwise there is nothing to prove. Given $z \notin$ $K \cup\left\{z_{0}\right\}$, there is a polynomial $p$ such that $p(z)=1>\|p\|_{K}$, by the polynomial convexity of $K$. Choose a polynomial $q$ such that $q\left(z_{0}\right)=0$ and $q(z)=1$. Let $M=\|q\|_{K}$, and choose an integer $n$ large enough so that $\left\|p^{n}\right\|_{K}<\frac{1}{2 M}$. Then $q(z) p^{n}(z)=1, q\left(z_{0}\right) p^{n}\left(z_{0}\right)=0$, and

$$
\max _{x \in K}\left|q(x) p^{n}(x)\right|<\frac{1}{2} .
$$

Thus $z$ does not belong to the polynomially convex hull of $K \cup\left\{z_{0}\right\}$.

For a compact set $K \subset \mathbf{C}^{n}$, in addition to the algebras $C(K), P(K)$ and $R(K)$ defined in the introduction, we define the algebra $A(K)$ as the closure in $C(K)$ of the set of functions holomorphic in a neighborhood (dependent on the function) of $K$. We have

$$
P(K) \subset R(K) \subset A(K) \subset C(K) .
$$

We will make use of the Oka-Weil Theorem (see [1], page 37), which states that if $\hat{K}=K$, then $A(K)=P(K)$. A compact set $K \subset \mathbf{C}^{n}$ is said to be holomorphically convex if $K$ is the intersection of domains of holomorphy; it is well known that polynomially convex sets are holomorphically convex (cf. [1], Lemma 7.4). 
We will have occasion to use two well-known results concerning the algebra $R(K)$, for $K$ a compact subset of the plane. First, $R(K)$ is local (see [4], Theorem 3.2.13): if a collection $\left\{U_{j}\right\}$ of open sets covers $K$, and $f$ is a continuous function on $K$ whose restriction to $\overline{U_{j}} \cap K$ lies in $R\left(\overline{U_{j}} \cap K\right)$ for each $j$, then $f \in R(K)$. Second, if $K \subset \mathbf{C}$ has two-dimensional Lebesgue measure zero, then $R(K)=C(K)$ (the Hartogs-Rosenthal Theorem - see 4], Theorem 3.2.4).

Another way to "localize" uniform algebras is by considering sets of antisymmetry. Given a compact set $K$, a subset $F$ of $K$ is said to be a set of antisymmetry for a uniform algebra $A$ on $K$ if whenever $g \in A$ and $g$ is real-valued on $F$, then the restriction of $g$ to $F$ is constant. The Bishop antisymmetric decomposition ([4, Theorem 2.7.5) states that $K$ is the disjoint union of the collection $\left\{F_{\alpha}\right\}$ of maximal sets of antisymmetry for $A$, that the restriction $A_{\alpha}$ of $A$ to each $F_{\alpha}$ is uniformly closed in $C\left(F_{\alpha}\right)$, and that a continuous function $g$ is in $A$ if and only if $\left.g\right|_{F_{\alpha}}$ is in $A_{\alpha}$ for each $\alpha$.

Let $M$ be a real submanifold of $\mathbf{C}^{n}$, of class $C^{1}$. We say that $M$ has a complex tangent at $p \in M$ if the real tangent space $T_{p} M$, identified with a real subspace of $\mathbf{C}^{n} \approx \mathbf{R}^{2 n}$, contains a nontrivial complex subspace of $\mathbf{C}^{n}$. The manifold $M$ is said to be totally real if it has no complex tangents.

Proposition 2.3. Let $K$ be a holomorphically convex compact set, and let $K_{0}$ be a compact subset of $K$ such that $K \backslash K_{0}$ is a totally real submanifold of $\mathbf{C}^{n}$, of class $C^{1}$. Then a continuous function $f$ belongs to $A(K)$ if and only if there exists $g \in A(K)$ with $f=g$ on $K_{0}$.

This result is due to O'Farrell, Preskenis and Walsh [10]. Under a stronger smoothness hypothesis on $M$ it was first proved by Hörmander and Wermer [7] who also reached the stronger conclusion that $f \in A(K)$ if and only if $\left.f\right|_{K_{0}} \in A\left(K_{0}\right)$. To establish Proposition 2.3, O'Farrell, Preskenis and Walsh reformulate the statement as an equivalent assertion concerning annihilating measures of the algebra $A(K)$ : if $\mu$ is a measure on $K$ such that

$$
\int f d \mu=0 \text { for all } f \in A(K)
$$

then $\mu$ is supported on $K_{0}$. This statement also follows from the HörmanderWermer result.

It is convenient to state the following corollary of Proposition 2.3:

Corollary 2.4. Let $K$ be a polynomially convex compact subset of $\mathbf{C}^{n}$, and let $K_{0}$ be a compact subset of $K$ such that $K \backslash K_{0}$ is a totally real submanifold of $\mathbf{C}^{n}$, of class $C^{1}$. If $P\left(K_{0}\right)=C\left(K_{0}\right)$, then $P(K)=C(K)$.

Proof. Let $\mu$ be a measure supported on $K$ with

$$
\int f d \mu=0 \text { for all } f \in P(K) .
$$

It suffices to show that $\mu$ is identically zero. By the Oka-Weil Theorem, and the remarks following Proposition 2.3, $\mu$ is supported on $K_{0}$. Let $\phi$ be an arbitrary continuous function on $K$. By hypothesis, we may choose a sequence $P_{n}$ of polynomials with $P_{n}$ converging uniformly to $\phi$ on $K_{0}$. Then

$$
\int_{K} \phi d \mu=\int_{K_{0}} \phi d \mu=\lim _{n \rightarrow \infty} \int_{K_{0}} P_{n} d \mu=0,
$$

the last equality following from (1). Hence $\mu$ is the zero measure. 
The following characterization of the points where a real submanifold $M$ has a complex tangent is no doubt well known. For an $m$-tuple $I=\left(i_{1}, \ldots, i_{m}\right)$ with $1 \leq i_{1}<\ldots<i_{m} \leq n$ we let $d z_{I}=d z_{i_{1}} \wedge \ldots \wedge d z_{i_{m}}$.

Lemma 2.5. Let $M$ be a real $m$-dimensional submanifold of $\mathbf{C}^{n}$, of class $C^{1}$. Then $M$ has a complex tangent at $p$ if and only if $d z_{I}(p)=0$ (as a form on $M$ ), for all $m$-tuples $I$.

Proof. Fix $p \in M$, and choose local coordinates $u_{1}, \ldots, u_{m}$ on $M$ in some neighborhood of $p$. The vectors $v_{j}=\left(\partial z_{1} / \partial u_{j}(p), \ldots, \partial z_{n} / \partial u_{j}(p)\right), 1 \leq j \leq m$ (considered as elements of $\mathbf{R}^{2 n}$ ), form a basis for the tangent space $T_{p} M$ over $\mathbf{R}$. Note that $M$ has a complex tangent at $p$ if and only if $\operatorname{dim}\left(T_{p} M \cap i T_{p} M\right)>0$. Since

$$
\operatorname{dim}\left(T_{p} M+i T_{p} M\right)=2 m-\operatorname{dim}\left(T_{p} M \cap i T_{p} M\right)
$$

it follows that $M$ has a complex tangent at $p$ if and only if the vectors $v_{1}, \ldots, v_{m}$ are linearly dependent over $\mathbf{C}$. The conclusion of the lemma follows by noting that $v_{1}, \ldots, v_{m}$ are linearly dependent over $\mathbf{C}$ if and only if the rank of the $n \times m$ matrix $J=\left(\left(\partial z_{l} / \partial u_{k}\right)\right)$ at $p$ is less than $m$, and that

$$
d z_{I}=\operatorname{det}\left(J_{I}\right) d u_{1} \wedge \ldots \wedge d u_{m}
$$

for every $m$-tuple $I=\left(i_{1}, \ldots, i_{m}\right)$, where $J_{I}$ is the $m \times m$ minor consisting of the rows $i_{1}, \ldots, i_{m}$ of $J$.

A relatively closed subset $V$ of an open set $O \subset \mathbf{C}^{n}$ is said to be a realanalytic subvariety of $O$ if for each $p \in V$ there exists a neighborhood $O^{\prime} \subset O$ of $p \in \mathbf{C}^{n}$ and real-valued functions $g_{1}, \ldots, g_{m}$ which are real-analytic in $O^{\prime}$, such that

$$
V \cap O^{\prime}=\left\{q \in O^{\prime}: g_{1}(q)=\cdots=g_{m}(q)=0\right\} .
$$

A point $p$ is a regular point of $V$ if there exists a neighborhood $U$ of $p$ in $\mathbf{C}^{n}$ such that $V \cap U$ is a real-analytic submanifold of $U$. The set of regular points will be denoted by $V_{\text {reg }}$. The dimension of $V$ as a manifold in a neighborhood of a regular point is constant on connected components of $V_{\text {reg }}$. The dimension of $V$ is defined to be the maximum of these dimensions over all connected components of $V_{\text {reg }}$. The set of singular points is defined to be $V_{\text {sing }} \equiv V \backslash V_{\text {reg }}$. Although $V_{\text {sing }}$ need not be a subvariety (unlike in the case of complex-analytic varieties), we have the following result on the Hausdorff measure of the singular set (see [5], 3.4.10, p. 337):

Lemma 2.6. Let $V$ be an m-dimensional real-analytic subvariety of an open set $O \subset \mathbf{C}^{n}$. Then the $m-1$ dimensional Hausdorff measure $\mathcal{H}^{m-1}\left(V_{\text {sing }} \cap C\right)$ is finite for each compact subset $C$ of $O$.

\section{Proof of Theorem 1.1}

We begin the proof of Theorem 1.1 with two lemmas which may be of independent interest.

Lemma 3.1. Let $Y \subset \mathbf{C}^{n}$ be a compact set such that $P(Y)=C(Y)$. Let $S \subset \mathbf{C}^{n}$ be a set with two-dimensional Hausdorff measure zero. Assume also that $Y \cup S$ is compact and polynomially convex. Then $P(Y \cup S)=C(Y \cup S)$.

The proof of Lemma 3.1 below is based on the proof of Theorem 30.1 (C) in [11]. 
Proof. It suffices to show that for every $z \in S \backslash Y$ and every $z^{\prime} \in Y \cup S$ with $z^{\prime} \neq z$, there is a real-valued function $f \in P(Y \cup S)$ such that $f(z) \neq f\left(z^{\prime}\right)$. To see this, note that each set of antisymmetry for $P(Y \cup S)$ then either consists of a single point or else is contained in $Y$. Consequently, if $g \in C(Y \cup S)$ and $F$ is a set of antisymmetry for $P(Y \cup S)$, then (since $P(Y)=C(Y)$ ) we have that $\left.g\right|_{F}$ is the uniform limit of functions in $\left.P(Y \cup S)\right|_{F}$. The Bishop antisymmetric decomposition (see section 2) then gives $g \in P(Y \cup S)$.

Fix $z \in S \backslash Y$ and $z^{\prime} \in Y \cup S, z \neq z^{\prime}$. Since $P(Y)=C(Y), Y$ is polynomially convex. By Lemma 2.2, $Y \cup\left\{z^{\prime}\right\}$ is also polynomially convex. Consequently there is a polynomial $p$ with $|p| \leq \frac{1}{2}$ on $Y \cup\left\{z^{\prime}\right\}$ and $p(z)=1$. It follows easily from the definition of Hausdorff measure that $p(S) \subset \mathbf{C}$ has two-dimensional Hausdorff measure zero, hence two-dimensional Lebesgue measure zero. By the HartogsRosenthal theorem, $R(K)=C(K)$ for each compact set $K \subset p(S)$. Consequently, if $h$ is a real-valued continuous function on $p(Y \cup S)$ with $h=0$ on $p(Y \cup S) \cap\{\zeta$ : $\left.|\zeta| \leq \frac{7}{8}\right\}$, and $h(1)=1$, then $h$ belongs to $R(p(Y \cup S))$ locally: $h$ clearly belongs to $R\left(p(Y \cup S) \cap\left\{\zeta:|\zeta| \leq \frac{7}{8}\right\}\right)$, and $h$ belongs to $R\left(p(Y \cup S) \cap\left\{\zeta:|\zeta| \geq \frac{3}{4}\right\}\right)$ as $p(Y \cup S) \cap\left\{\zeta:|\zeta| \geq \frac{3}{4}\right\}$ is a compact subset of $p(S)$. Since $R(K)$ is a local algebra for $K \subset \mathbf{C}$, we have $h \in R(p(Y \cup S))$. Hence $h \circ p \in R(Y \cup S)$, $h \circ p$ is real-valued, and $h \circ p(z) \neq h \circ p\left(z^{\prime}\right)$. Moreover, since $Y \cup S$ is assumed polynomially convex, the Oka-Weil Theorem implies $R(Y \cup S)=P(Y \cup S)$, so $h \circ p \in P(Y \cup S)$. This completes the proof.

Lemma 3.2. Let $M$ be a real $m$-dimensional submanifold of $\mathbf{C}^{n}$, of class $C^{1}$. Suppose that $K$ is a compact subset of $M$ satisfying (i) and (ii). Let $E$ be the set of points at which $M$ has a complex tangent. Then $E \cap K$ has empty interior in $M$.

Proof. By Lemma 2.5, it suffices to show that given any set $U \subset K$ which is open in $M$, we can choose an $m$-tuple $I=\left(i_{1}, \ldots, i_{m}\right)$ such that $d z_{I}$ is not identically zero as a form on $U$. We will choose $i_{1}, \ldots, i_{m}$ inductively so that for each $k$, $1 \leq k \leq m, d z_{i_{1}} \wedge \ldots \wedge d z_{i_{k}}$ is not identically zero on $U$. This can clearly be done when $k=1$, since the functions $z_{1}, \ldots, z_{n}$ separate points on $U$. Now assume that a $k$-tuple $I^{\prime}=\left(i_{1}, \ldots, i_{k}\right)$ has been found so that $d z_{I^{\prime}}$ does not vanish identically on $U$. Choose a point $p \in U$ such that $d z_{I^{\prime}}(p) \neq 0$ and $k$ linearly independent vectors $\xi_{1}, \ldots, \xi_{k}$ in the tangent space to $M$ at $p$ such that $d z_{I^{\prime}}(p)\left(\xi_{1}, \ldots, \xi_{k}\right) \neq 0$. We may then choose a set $N \subset U$, homeomorphic to a $(k+1)$-ball, with $p \in \partial N$, such that $\left(\xi_{1}, \ldots, \xi_{k}\right)$ span the tangent space to $\partial N$ at $p$. Then $d z_{I^{\prime}}$ does not vanish identically as a form on $\partial N$. By standard theorems there is a smooth function $g$ on $\partial N$ so that

$$
\int_{\partial N} g d z_{I^{\prime}} \neq 0
$$

Letting $K_{0}=\left\{p \in \partial N: d z_{I^{\prime}}(p)=0\right.$ as a form on $\left.\partial N\right\}$, we may assume that $g$ vanishes on $K_{0}$. By Lemma 2.5, $\partial N \backslash K_{0}$ is a totally real submanifold of $\mathbf{C}^{n}$. Furthermore, by Lemma $2.1, \partial N$ is polynomially convex. We can therefore apply Proposition 2.3 with $K=\partial N$. Since $g$ vanishes on $K_{0}$, the restriction of $g$ to $K_{0}$ lies in $A\left(K_{0}\right)$. By Proposition 2.3, then, $g \in A(\partial N)$. The Oka-Weil Theorem then implies $g \in P(\partial N)$. We can therefore choose the function $g$ satsifying (2) to be a polynomial. By Stokes' Theorem,

$$
\int_{N} d g \wedge d z_{I^{\prime}}=\int_{\partial N} g d z_{I^{\prime}} \neq 0
$$


The form $d g \wedge d z_{I^{\prime}}$ is a linear combination (the coefficients of which are smooth functions) of forms of the type $d z_{I^{\prime}} \wedge d z_{l}$. By (3), at least one of these is not identically zero on $U$, which completes the induction and the proof.

Lemma 3.2, whose proof is similar to that of Theorem 2.1 of [6], can also be established in the case when $M$ is of class $C^{2}$ by using the fact that the interior of $E \cap K$ must contain the boundary of an analytic disk (as can be shown using [1], Theorem 18.7), which would violate one of the conditions (i) or (ii).

Now to prove Theorem 1.1, let $E$ denote the set of points at which $\Sigma$ has a complex tangent, $X_{0}$ the interior of $X$ relative to $\Sigma$, and let $\Omega_{0}$ be an open subset of $\mathbf{C}^{n}$ with $X_{0}=X \cap \Omega_{0}$. Let $\tilde{E}=E \cap X_{0}$, and $K_{0}=\partial X \cup \tilde{E}$. Since each limit point of $\tilde{E}$, which is not itself in $\tilde{E}$, belongs to $\partial X$, we see that $K_{0}$ is compact. Since $X$ is polynomially convex by assumption, and $X \backslash K_{0}$ is a totally real submanifold of $\Omega_{0}$, Corollary 2.4 implies that in order to prove $P(X)=C(X)$ it suffices to show that $P\left(K_{0}\right)=C\left(K_{0}\right)$.

Let $\tilde{E}_{c}$ denote the points at which $\tilde{E}_{r e g}$ itself has a complex tangent. Set $Z=$ $\partial X \cup \tilde{E}_{\text {sing }} \cup \tilde{E}_{c}$. It is not hard to verify that $Z$ is compact and that $K_{0} \backslash Z$ is a totally real, real-analytic submanifold of $\Omega_{0}$. By Lemma $2.1, K_{0}$ is polynomially convex, and so Corollary 2.4 implies that to show $P\left(K_{0}\right)=C\left(K_{0}\right)$ it suffices to show that $P(Z)=C(Z)$. To do this, we will apply Lemma 3.1, taking $Y=\partial X$ and $S=\tilde{E}_{\text {sing }} \cup \tilde{E}_{c}$. Note that since $X$ satisfies (i) and (ii), Lemma 2.1 shows that $\partial X$ also satisfies (i) and (ii). Since $\partial X$ is a two-manifold, the peak point theorem for two-manifolds [2] shows that $P(\partial X)=C(\partial X)$. Moreover, Lemma 2.1 implies that $Z$ is polynomially convex. Thus by Lemma 3.1 to show that $P(Z)=C(Z)$ and complete the proof of Theorem 1.1, it suffices to show that $\tilde{E}_{\text {sing }} \cup \tilde{E}_{c}$ has two-dimensional Hausdorff measure zero.

It follows easily from Lemma 2.5 that $\tilde{E}$ is a real-analytic subvariety of $\Omega_{0}$. By Lemma $3.2, \tilde{E}$ has no interior in $\Sigma$; hence the dimension of $\tilde{E}$ is at most two. We can now show that $\tilde{E}_{\text {sing }}$ and $\tilde{E}_{c}$ each have two-dimensional Hausdorff measure zero. First, consider $\tilde{E}_{\text {sing }}$. Since the dimension of $\tilde{E}$ is at most two, Lemma 2.6 implies for each compact set $C \subset \Omega_{0}, \mathcal{H}^{1}\left(C \cap \tilde{E}_{\text {sing }}\right)<\infty$. Exhausting $\Omega_{0}$ by a countable family of compact sets, we see that $\mathcal{H}^{2}\left(\tilde{E}_{\text {sing }}\right)=0$. Next, let $K$ be an arbitrary compact subset of $\tilde{E}_{\text {reg }}$. By Lemma $2.1, K$ satisfies (i) and (ii). Lemma 3.2 shows that $\tilde{E}_{c} \cap K$ has no interior relative to $\tilde{E}_{r e g}$, from which it follows that $\tilde{E}_{c}$ is a real-analytic subvariety of $\Omega_{0}$ of dimension at most 1 . Thus $\mathcal{H}^{2}\left(\tilde{E}_{c}\right)=0$, and the proof is complete.

\section{REFERENCES}

[1] H. Alexander and J. Wermer, Several Complex Variables and Banach Algebras, Third edition, Springer, 1998. MR 98g:32002

[2] J. T. Anderson and A. J. Izzo, A Peak Point Theorem for Uniform Algebras Generated by Smooth Functions On a Two-Manifold, Bull. London Math. Soc. (to appear)

[3] R. F. Basener, On Rationally Convex Hulls, Trans. Amer. Math. Soc. 182 (1973), pp. 353381. MR 52:803

[4] A. Browder, Introduction to Function Algebras, Benjamin, New York, 1969. MR 39:7431

[5] H. Federer, Geometric Measure Theory, Springer, 1969. MR 41:1976

[6] M. Freeman, Some Conditions for Uniform Approximation on a Manifold, in: Function Algebras, F. Birtel (ed.), Scott, Foresman and Co., Chicago, 1966, pp. 42-60. MR 33:1758

[7] L. Hörmander and J. Wermer, Uniform Approximation on Compact Subsets in $\mathbf{C}^{n}$, Math. Scand. 23 (1968), pp. 5-21. MR 40:7484 
[8] A. J. Izzo, Failure of Polynomial Approximation on Polynomially Convex Subsets of the Sphere, Bull. London Math. Soc. 28 (1996), pp. 393-397. MR 98d:32017

[9] R. Nirenberg and R. O. Wells, Approximation Theorems on Differentiable Submanifolds of a Complex Manifold, Trans. Amer. Math. Soc. 142 (1969), pp. 15-35. MR 39:7140

[10] A. J. O'Farrell, K. J. Preskenis, and D. Walsh, Holomorphic Approximation in Lipschitz Norms, in Proceedings of the Conference on Banach Algebras and Several Complex Variables, Contemporary Math. v. 32, American Mathematical Society, 1983. MR 86c:32015]

[11] E.L. Stout, The Theory of Uniform Algebras, Bogden and Quigley, 1971. MR 54:11066]

[12] J. Wermer, Polynomially Convex Disks, Math. Ann. 158 (1965), pp. 6-10. MR 30:5158

Department of Mathematics, College of the Holy Cross, Worcester, Massachusetts 01610

E-mail address: anderson@math.holycross.edu

Department of Mathematics and Statistics, Bowling Green State University, BowlING Green, Ohio 43403

E-mail address: aizzo@math.bgsu.edu

Current address: Department of Mathematics, Texas A \& M University, College Station, Texas 77843

E-mail address: aizzo@math.tamu.edu

Department of Mathematics, Brown University, Providence, Rhode Island 02912

E-mail address: wermer@math.brown.edu 